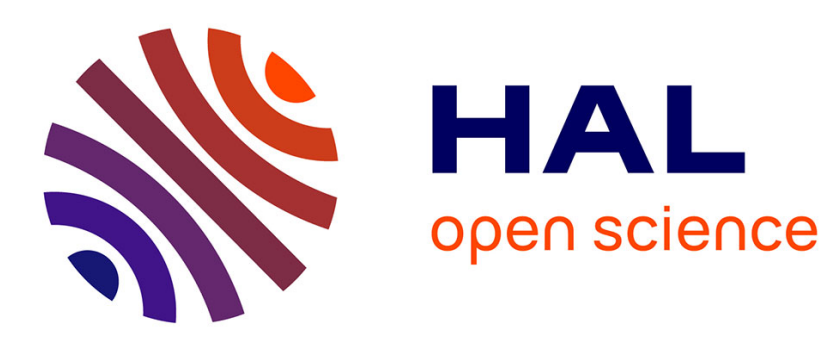

\title{
Electron microscopy study of the modulated phases in berlinite AlPO4 and quartz
}

\author{
Etienne Snoeck, Christian Roucau, P. Saint-Grégoire
}

\section{To cite this version:}

Etienne Snoeck, Christian Roucau, P. Saint-Grégoire. Electron microscopy study of the modulated phases in berlinite AlPO4 and quartz. Journal de Physique, 1986, 47 (12), pp.2041-2053. 10.1051/jphys:0198600470120204100 . jpa-00210399

\section{HAL Id: jpa-00210399 https://hal.science/jpa-00210399}

Submitted on 1 Jan 1986

HAL is a multi-disciplinary open access archive for the deposit and dissemination of scientific research documents, whether they are published or not. The documents may come from teaching and research institutions in France or abroad, or from public or private research centers.
L'archive ouverte pluridisciplinaire HAL, est destinée au dépôt et à la diffusion de documents scientifiques de niveau recherche, publiés ou non, émanant des établissements d'enseignement et de recherche français ou étrangers, des laboratoires publics ou privés. 
Classification

Physics Abstracts

$61.14-64.70 \mathrm{~K}$

\title{
Electron microscopy study of the modulated phases in berlinite $\mathrm{AlPO}_{4}$ and quartz
}

\author{
E. Snoeck, C. Roucau and P. Saint-Grégoire (*) \\ Laboratoire d'Optique Electronique du CNRS, 29, rue J. Marvig, 31055 Toulouse Cedex, France \\ (*) G.D.P.C. (LA CNRS n 233). U.S.T.L., 34060 Montpellier Cedex, France
}

(Reçu le 7 avril 1986, accepté sous forme définitive le 23 août 1986)

\begin{abstract}
Résumé. - La séquence des transitions de phase $\beta \rightarrow$ phase incommensurable $\rightarrow \alpha$ de $\mathrm{AlPO}_{4}$ et de $\mathrm{SiO}_{2}$ est étudiée en microscopie électronique. Les caractéristiques de la phase modulée sont en accord avec les résultats déjà obtenus en diffraction des neutrons. Comme prévu par la théorie, une désorientation du vecteur d'onde vis-à-vis des directions $O Y$ a été observée. Nous présentons la variation en température de cet angle et celle-ci nous permet d'étudier la variation de la largeur des discommensurations. Cela semble confirmer l'hypothèse d'un changement continu de la modulation d'un régime sinusoïdal proche de $T_{\mathrm{i}}$ vers une structure en domaine lorsque la température décroît (ce changement de régime est aussi confirmé par nos études en diffraction électronique qui montrent l'apparition de taches satellites d'ordre supérieur pour ces températures). Des défauts intrinsèques de la phase incommensurable ont été mis en évidence. Ceux-ci correspondent à des imperfections locales de la période de la modulation nécessaires pour faire varier le vecteur d'onde. Dans des échantillons impurs d'AlPO ${ }_{4}$, un piégeage de ces défauts intrinsèques par des défauts propres au cristal a été observé. Ceci explique les études menées en biréfringence sur ce type d'échantillon, suggérant une variation discontinue du vecteur d'onde $\mathbf{k}$. La transition de blocage vers la phase $\alpha$ entraine l'apparition d'un état intermédiaire, constitué de microdomaines en forme de triangles allongés. Un modèle utilisant des défauts particuliers de la phase modulée est proposé pour expliquer la nucléation de cette phase intermédiaire. Nos travaux sur la dynamique (et sur la relaxation) de la transition de phase $\alpha$ /phase incom/ $\beta$ sont aussi présentés et étudiés qualitativement. Dans un dernier temps, nous donnons une interprétation possible de l'effet mémoire et du phénomène de relaxation (basé sur la présence de défauts intrinsèques), qui diffère de celle de Jamet et Lederer.
\end{abstract}

\begin{abstract}
The sequence of $\beta \rightarrow$ incommensurate (I) $\rightarrow \alpha$ phases taking place in $\mathrm{AlPO}_{4}$ and $\mathrm{SiO}_{2}$ is studied by means of electron microscopy. The features of the modulated phase are in agreement with previous neutron determination of incommensurability, and as predicted by theory, the modulation wave vector is slightly rotated from the $O Y$ directions ; the thermal variation of this angle is given, and this data allows an approximate estimation of the change of discommensuration width that confirms the scheme of a gradual change from a sinusoidal modulation, close to $T_{\mathrm{i}}$, to a domain-like structure when $\left(T_{\mathrm{i}}-T\right)$ increases (this is also confirmed by electron diffraction data showing appearance of higher order satellites). The incommensurate phase is marked by the presence of intrinsic defects corresponding to local imperfections of the superperiod, which are involved in the k-variation. In impure $\mathrm{AlPO}_{4}$ samples, a pinning of intrinsic defects by imperfections of the crystal lattice occurs. This explains the birefringence behaviour suggesting a discontinuous variation of $\mathbf{k}$. The (lock-in) transition to the $\alpha$ phase involves an intermediate state characterized by elongated triangular microdomains, and proceeds by nucleation ; a model implying some particular defects is proposed. Dynamical (and relaxation) phenomena are also reported and qualitatively interpreted. Finally, we give a possible interpretation of memory effect and of relaxation phenomena based on intrinsic defects which differs from that of Jamet and Lederer.
\end{abstract}

\section{Introduction.}

Aluminum phosphate is a quartz analog, the structure of which can be visualized roughly by replacing one $\mathrm{Si}^{4+}$ ion in $\mathrm{SiO}_{2}$ by $\mathrm{Al}^{3+}$ and the other by $\mathrm{P}^{5+\cdot}$; the cell is then approximately doubled in the $c$ direction. Like quartz, $\mathrm{AlPO}_{4}$ presents an $\alpha \rightarrow \beta$ structural change, from a hexagonal $\left(D_{6}^{4}\right)$ to a trigonal phase $\left(D_{3}^{4}\right)$, and the studies performed up to now in both compounds are in favour with a same mechanism for the structural changes [1-3]. The 
order parameter (O.P.) $\eta$ of the transition is a single component quantity, transforming as the $B_{1}$ representation of the $\beta$ phase group, and which can be considered as the angle of rotation of the $\mathrm{SiO}_{4}$ (or $\mathrm{AlO}_{4}$ and $\mathrm{PO}_{4}$ ) tetrahedra around their twofold axis. Attention has been drawn on these compounds by Aslanyan and Levanyuk [4], after an electron microscopy study by Van Tendeloo et al. [5] : between the $\alpha$ and $\beta$ phases, an inhomogeneous state was observed, corresponding to a pattern of triangular equilateral microdomains. Moreover, an inhomogeneous state had also been reported some years before in quartz, between both phases : light scattering experiments revealed static inhomogeneities of the order of the $\mu \mathrm{m}[6,7]$. The O.P. being a one-dimensional quantity, the Lifchitz criterion is necessarily satisfied, but the presence of an incommensurate phase is possible if there is a coupling between the O.P. and another degree of freedom ; in the case of sodium nitrite $\mathrm{NaNO}_{2}$, for example, the presence of a modulated phase can be attributed to a Lifchitz-like invariant [8]. For the present $\alpha \rightarrow \beta$ transitions, an invariant coupling the spatial gradient of $\eta$ with some components of the strain tensor can induce an incommensurate phase, whose characteristics are in agreement with the results of Van Tendeloo et al. [5]. Indeed, the modulation wave vector is predicted to lie in the direction of the twofold axes which are lost in the $\alpha$ phase, and this explains the triangular shape of the observed microdomains; let us note however that up to now, the research of satellites by electron diffraction was unsuccessful. The validity of theory has been verified first in quartz : birefringence measurements revealed a discrete anomaly [9], corresponding to a supplementary transition. Some time after, the incommensurate character of the intermediate phase was demonstrated by neutron diffraction [10].

In $\mathrm{AlPO}_{4}$, recent experiments [11] also showed an intermediate modulated phase analogous to that of quartz, but which extends on a wider temperature range. The crystals studied are artificial, and the anomalies which were observed in the specific heat and birefringence measurements, depend strongly on the growth sector, the sample, and the thermal history [11-13]. The aim of this paper is to present the results of the electron microscopy study we performed with various samples in order to understand the phenomena which occur in the incommensurate phase of this compound, and the sensitivity on defects. The modulated phase of quartz is also investigated. Our study is complementary to those of Van Tendeloo et al. [5], Van Landuyt et al. [14], and Yamamoto et al. [15].

In section 2 , we recall the characteristics of the modulated phase in $\mathrm{AlPO}_{4}$, and the main features of previously obtained results interesting here. Section 3 is devoted to the results obtained with the electron microscope, giving, in particular, information on the intrinsic defects of the incommensurate structure ; finally the conclusion is given in section 4 .

\section{The incommensurate phase of berlinite.}

The expansion of the free energy density, including the O.P., its relevant spatial derivatives, and the elastic strains $u_{i j}$, takes on the form [16] :

$$
\begin{aligned}
f(\mathbf{r})= & f_{0}+\frac{A}{2} \eta^{2}+\frac{B}{4} \eta^{4}+\frac{g}{2}(\nabla \eta)^{2}+\frac{h}{2}(\Delta \eta)^{2}+G\left[\left(\frac{\partial \eta}{\partial x}\right)^{3}-3 \frac{\partial \eta}{\partial x}\left(\frac{\partial \eta}{\partial y}\right)^{2}\right]+ \\
& +\frac{1}{2} \sum_{i, j, k, l} c_{i j k l} u_{i j} u_{k l}+\frac{a}{2}\left[\left(u_{11}-u_{22}\right) \frac{\partial \eta}{\partial x}-2 u_{12} \frac{\partial \eta}{\partial y}\right]+r_{1} \eta^{2}\left(u_{11}+u_{22}\right)+r_{3} \eta^{2} u_{33}+\cdots
\end{aligned}
$$

and the thermodynamic potential is : $F=\int_{V} f(\mathbf{r}) \mathrm{d}^{3} r$.

In this expression, $x$ corresponds to the twofold axis which is conserved in the $\alpha$ phase, and $y$ to the one which is lost.

As usually, the coefficient $A$ is written $A=a_{0}\left(T-T_{0}\right)$, with $a_{0}>0$; the invariant with the coefficient $a / 2$ is responsible for the modulation of $\eta$. Indeed, by eliminating the strains and introducing the Fourier components of $\eta$, one obtains the expression, with « renormalized» coefficients :

$$
F=F_{0}+\sum_{\mathbf{k}} \frac{1}{2}\left\{A+\left[g-\frac{a^{2}}{4}\left(\frac{\cos ^{2} 3 \varphi}{C_{11}}+\frac{\sin ^{2} 3 \varphi}{C_{66}}\right)\right] \cdot k^{2}\right\} \eta_{\mathbf{k}} \eta_{-\mathbf{k}}+\cdots
$$

where $\varphi$ is the angle between $\mathbf{k}$ and the $O X$ axis.

The instability occurs at

$$
T_{\mathrm{i}}=T_{0}+\frac{\left(g-a^{2} / C_{66}\right)^{2}}{4 a_{0} h},
$$

for displacements modulated in three directions, namely that of the twofold axes which are lost in the $\alpha$ phase $\left(\varphi=\frac{\pi}{2}+\eta \frac{\pi}{3}\right)$. In [16], various possibilities for the structure of the I phase have been examined. If the modulations with the three vectors at $120^{\circ}$ are superimposed (and the presence of equilateral microdomains shows that it is actually the 
case), the cubic term corresponding to the invariant with $G / 3$, written in the Fourier space, does not vanish : its presence provokes a deviation of the $\mathbf{k}$ vector from the crystallographic $\langle O Y\rangle$ directions. This prediction has been verified in $\mathrm{SiO}_{2}$, by X-ray diffraction [17], and in our present work.

In $\mathrm{AlPO}_{4}$, the satellites are found roughly along the axis of the $\langle O Y\rangle$ type, but the deviation of $\mathbf{k}$ from the pure crystallographic directions was not searched in the neutron diffraction experiment of reference [11]. Systematic extinctions of the satellite occur, and can be well accounted for by symmetry arguments : according to theory, the atomic displacements in the I phase have two components, which are both expected to lead to the vanishing of the structure factors of satellites situated along $a^{*}$, around Bragg spots of the $h 00$ type. The position $\mathbf{k}$ of the satellites is, within experimental accuracy, the same for the three directions. On cooling from the $\beta$ phase, $k$ presents first a plateau on the value $k=3 \times 10^{-2} a^{*}$, and further decreases strongly to zero (Fig. 1). The I phase of berlinite has some remarkable features . In particular, the behaviour of the satellite width in the $\mathbf{k}$ direction is somewhat unusual, in the sense that on cooling, it increases well above the lock-in transition to the $\alpha$-phase (in contradiction with $\mathrm{SiO}_{2}$ where the increase occurs in the vicinity of $\left.T_{\mathrm{c}}[10]\right)$. Moreover, birefringence and specific heat measurements have provided interesting information ; in samples containing defects, the I phase is marked by the presence of a lot of anomalies : small jumps of $\Delta n$, and $C_{\mathrm{p}}$ peaks. Each peak of specific heat can be unambiguously related to a birefringence discontinuity, and this shows that one deals with states having a spatial extension in the crystal. Hysteresis phenomena were studied in detail by means of $\Delta n$ measurements : the lock-in transition at $T_{\mathrm{c}}$ has a clear first order character, as evidenced

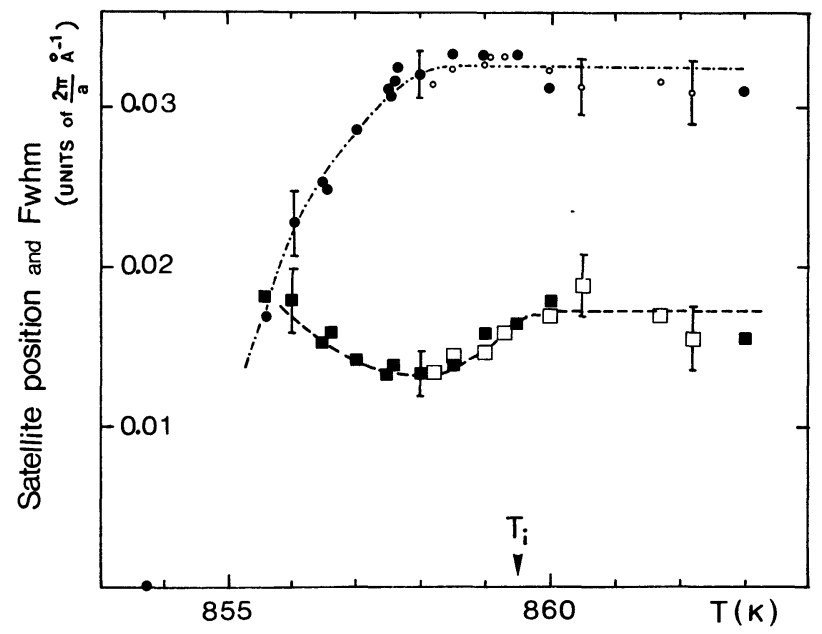

Fig. 1. - Thermal evolution of the satellite position (circles) and width FWHM (squares) in an $\mathrm{AlPO}_{4} \mathrm{~A}$ sample (neutron diffraction results from Ref. [11]). Black symbols refer to measurements on cooling, and white ones on heating. both by a jump and a thermal hysteresis. Above $T_{c}$, the hysteresis loop is anomalous, as there is a tail on the $\beta$-phase side : the reversibility occurs only approximately $3{ }^{\circ} \mathrm{C}$ on heating (Fig. 2). In the intermediate phase, a global hysteresis is observed; particular attention has been paid to the phenomena which are related to the discontinuities : between two small jumps, the birefringence variation is seen to be rigourously reversible, and tilted plateaux on the left branch of the hysteresis loop can be produced and identified to the plateau existing on the right main branch (Fig. 3). These data were interpreted in [13], as a manifestation of well defined intermediate states (characterized by an absence of hysteresis) associated with a constant given value of the wave vector $\mathbf{k}$, due to a pinning of the modulation by extrinsic defects.

The better samples, the more discrete discontinuities on the birefringence curve, and the smaller

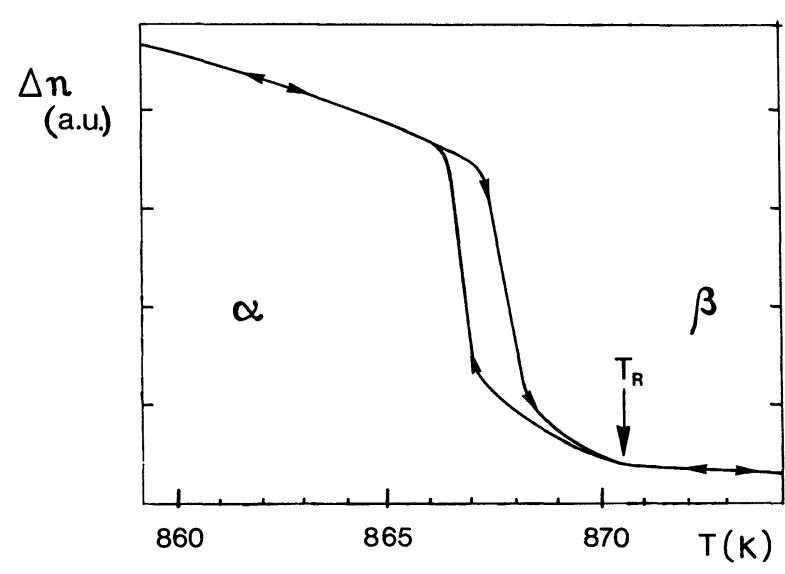

Fig. 2. - Linear birefringence as a function of temperature at the $\alpha-\beta$ transition in a berlinite B-sample (see Ref. [13]). $T_{\mathrm{R}}$ is the temperature above which reversibility occurs again ; it can be identified to the $\beta \rightarrow I$ transition temperature.

$\Delta n$

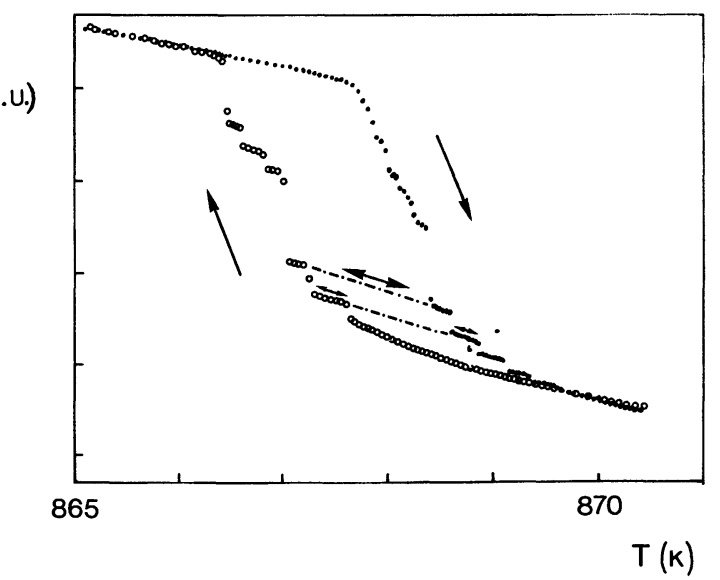

Fig. 3. - Linear birefringence as a function of temperature in a berlinite A-sample. The tilted plateaux are reversible, as indicated by the arrows which show the sense of the temperature variation. 
additional specific heat peaks : the crystals of berlinite which present clearly marked intermediate states, as shown in figure 3 , become somewhat milky when heated ; they will be referred to as « samples of A-type » in this paper. They correspond typically to crystalline germs synthetized in a medium of phosphoric acid (or phosphoric and chlorhydric acids) by the hydrothermal method. The B samples (with which results like those shown in figure 2 are obtained) are much closer to perfection, they originate from growth sectors of crystals realized in a medium of phosphoric acid ; the synthesis of berlinite has been in progress these last years, and the present samples of this type show no manifestation of pinning.

\section{Experiments.}

The original aim of our electron microscopy study, was mainly to clarify the difference of behaviour of the samples, and the evolution mechanism of the wavevector in the I phase and at the lock-in. For this reason a peculiar attention was paid to obtain good temperature homogeneities in the investigated part of the crystal, and a correct temperature stability. The samples were placed in a holder heated with a resistance in which the current was regulated from the indication of a PtRh-Pt thermocouple. Under these conditions, the temperature stability can be estimated to be approximately $1 / 10{ }^{\circ} \mathrm{C}$. The thicknesses necessary to work (around $100 \mathrm{~nm}$ ) have been obtained either by fracture of the specimens, or by ion thinning with a grazing beam. For the electron diffraction experiments, we mainly used a Philips EM $400(120 \mathrm{kV})$, whereas the images were generally obtained with a Jeol $200 \mathrm{CX}(200 \mathrm{kV})$, using a video recorder (for this reason the pictures are sometimes slightly distorted) to get information on dynamics.

\subsection{IMAGES OBTAINED WITH AlPO $_{4}$ CRYSTALS OF} THE A-TYPE. - In these samples (in which strong pinning effects of the modulation are expected) we mainly performed experiments with the hexagonal axis of the crystal perpendicular to the electron beam. In this geometry, the incommensurate phase appears as striped : along the three directions corresponding to the $\mathrm{OY}$ axis, the O.P. $\eta$ of the $\beta \rightarrow \alpha$ transition is modulated, taking positive and negative values. According to the generally admitted scheme, when the lock-in transition is approached, the modulation is no longer sinusoidal, and a domain-like structure takes place gradually. This feature is qualitatively verified since the contrast of the fringes becomes more pronounced close to $T_{\text {c }}$.

The absolute determination of temperatures in electron microscopy is a difficult task, since the thermocouple is not situated on the sample itself. In this experiment, we maintained the intensity of the beam, constant and as low as possible : the relative values of $T$ are then expected to be correct. In figure 4 , one sees the pictures obtained at four decreasing temperatures ; in (a), the crystal enters
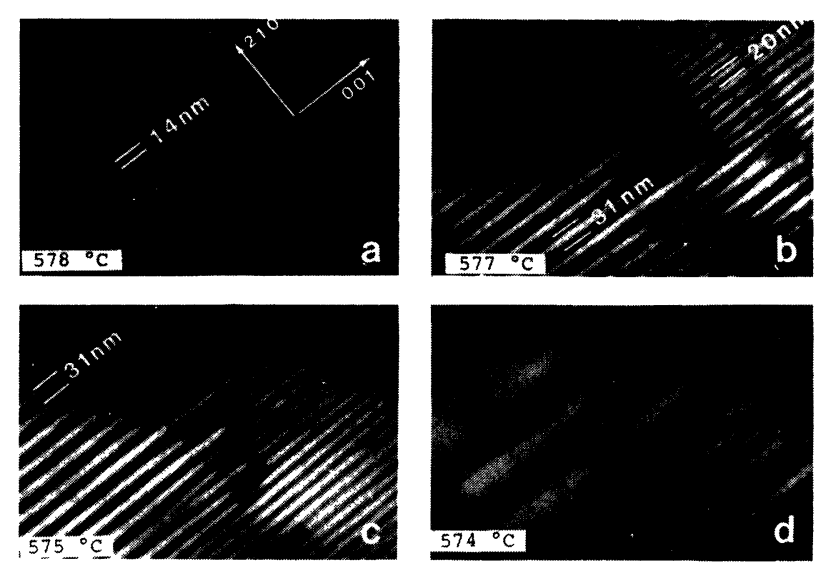

Fig. 4. - Dark field images in the I-phase obtained with $107 \mathrm{Bragg}$ spot in an $\mathrm{AlPO}_{4} \mathrm{~A}$-sample at various temperatures. Note the presence of deperiodization defects. The image at $574{ }^{\circ} \mathrm{C}$ corresponds to the onset of the $\alpha$-phase.

the I phase, and in (d) it begins to be in the $\alpha$ phase. The difference of temperatures is consistent with the birefringence results of figure 3 , obtained on the same crystal, and the value of $14 \mathrm{~nm}$ measured on the domain pattern is in good agreement with the neutron diffraction determination (see Fig. 1). On cooling, the size of the microdomains is stable in a range somewhat smaller than $1{ }^{\circ} \mathrm{C}$, and then increases : at $577^{\circ} \mathrm{C}$, an interesting phenomenon occurs, since two periodicities can be evidenced, namely 20 and $30 \mathrm{~nm}$. The junction between both regions is possibly realized by defects annihilating a microdomain $\left({ }^{1}\right)$. It can be seen that this state is stable in a range of at least $2{ }^{\circ} \mathrm{C}$, as at $575^{\circ} \mathrm{C}$ it still holds. The relative volume of the crystal modulated by different wave vectors $k$ does however vary with temperature.

it seems that the defects which we encounter here are the analogs of those described by Janovec [18, 19], and Prelovsek [20] for the cases of $\left(\mathrm{NH}_{4}\right)_{2} \mathrm{BeF}_{4}$ and $\mathrm{Rb}_{2} \mathrm{ZnCl}_{4}$. The mechanism for the $\mathbf{k}$ variation involves a motion along $\mathrm{OZ}$, of the deperiodization point defects $\mathrm{P}$ (see Fig. 5), which can in turn be pinned in certain temperature intervals, by defects of the crystal lattice. During cooling or heating, one observes actually an irregular motion of these objects. In figure 4 for example, where the « front » of $\mathbf{P}$ defects is visible, one observes it to be situated in the vicinity of a defect of the crystal (indicated by an arrow in picture 4-d) ; it separates the well defined regions with different periodicities. It can be noted that the values encountered are mutually commensurate: $k_{\mathrm{i}}$ being the value of $k$ close to $T_{\mathrm{i}}$ (corresponding to the periodicity of $14 \mathrm{~nm}$ ), the ratios $k_{\mathrm{i}} / k$ are $1,2 / 3,4 / 9$ within experimental accuracy.

( ${ }^{1}$ ) As the present study is performed on thin samples, it does not give precise information on the phenomena that occur in the bulk of bigger crystals. This is why we do not give any three dimensional model for the situation observed in figure 4. 


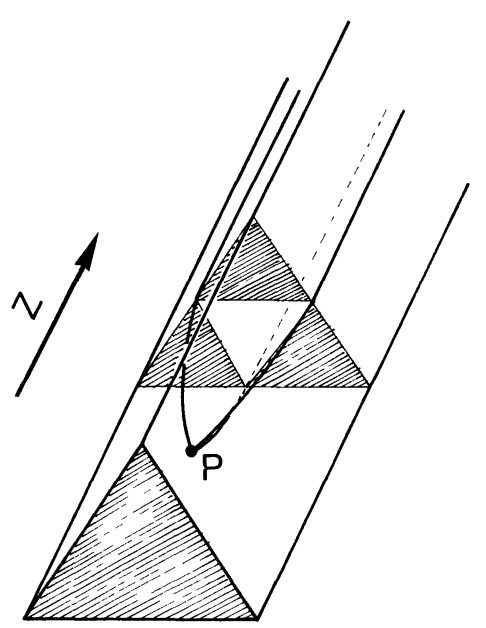

Fig. 5. - A deperiodization point defect P. Three edges of a microdomain meet at the P-point. Hatched and white triangles are characterized by opposite values of the O.P. $\eta$.

These observations allow us to specify the interpretation of the phenomena observed in these samples. The tilted plateau's joining the main branches of the hysteresis loop (see Fig. 3) of $\Delta n$, can now be related with the states where the $P$ defects remain pinned by defects of the crystal lattice. In this case, there is a coexistence, in well separated parts of the sample, of different $k$-values which remain constant in the temperature range defined by the plateau. It is therefore natural that the birefringence curve is then exactly reversible. When the temperature is changed so that it lies out of the limit of a plateau, the discarding with respect to equilibrium provokes a sudden depinning of the $\mathrm{P}$ defects, which results in a jump of $\Delta n$. These data are not in contradiction with the neutron diffraction results showing a continuous decrease of $k$ in the incommensurate phase : a change of $k$ by jumps on a finite number of values, with just a variation of the volume modulated by a given vector $\mathbf{k}$, leads to the existence of different peaks at fixed incommensurate positions in the reciprocal space, but with varying relative intensities. If the satellites are not resolved, one measures therefore a continuous change of $k$, corresponding to the displacement of the barycenter of the " satellite ", and an increase of their width (FWHM) due to the multi- $k$ state. These features are precisely observed in the temperature range where $k$ is seen to vary discontinuously by electron microscopy, and where the accidents occur on the birefringence curve.

The mechanism involving the $\mathrm{P}$ defects in the $k$ variation can be accounted for, if the frontal motion of the DC's is hindered, for example by impurities in the crystal. In this case, the value of $k$ at a given temperature $T$, is the one which would correspond (in a crystal where the frontal motion of DC's is free) to the thermodynamic equilibrium at $T^{\prime}>T$. Consequently the $k$-value occurring at $T$ does not minimize the free energy $F$, and if the energy of the $\mathrm{P}$ defect is smaller than the gain obtained by changing $k$, this object is created and the new state is more stable. The enhancement of hysteresis, which is clearly observed in the A samples, can be naturally attributed to the pinning of the $\mathrm{P}$ defects during their motion in the $\mathrm{OZ}$-direction.

It is worth giving a comment on the birefringence variation within the hysteresis cycle. As it is confirmesd here, the tilted plateau's represent a birefringence variation at constant $\mathbf{k}$. Their slope varies slightly in the hysteresis loop. Golovko and Levanyuk, considering the problem of the propagation of light in a modulated medium, concluded that the spontaneous variation of $\Delta n$ could depend both on the amplitude $\rho$ and the wave vector $\mathbf{k}$ of the O.P. [21]. Recent investigations by Fousek and Kroupa in $\mathrm{K}_{2} \mathrm{SeO}_{4}$ have evidenced a complex behaviour of $\Delta n$ [22], but no dependence on the discommensuration density.

In general, the birefringence change $\Delta n(\rho, \mathbf{k})$ can be therefore expanded as :

$$
\delta \Delta n \simeq \frac{\partial \Delta n}{\partial \rho} \delta \rho+\frac{\partial \Delta n}{\partial k} \delta k
$$

The first term in (3) describes the linear variation of the birefringence between the main branches. The discarding from linearity observed during a complete cooling or heating run can possibly be attributed to the $k$-variation. The small $\Delta n$ jumps would then express the $k$-discontinuities when depinning occurs, and possibly also a small change of $\rho$. The birefringence behaviour will be analysed elsewhere in detail, but it is interesting already to note the complex behaviour of this quantity, in agreement with theory.

\subsection{STUDY OF THE B-SAMPLES OF BERLINITE AND OF QUARTZ.}

3.2.1 Electron diffraction. - The diffraction patterns obtained at various temperatures are presented in figure 6 . The wavevectors of the modulation being directed along the OY-type axis, six satellites are generally observed around the Bragg spots. In
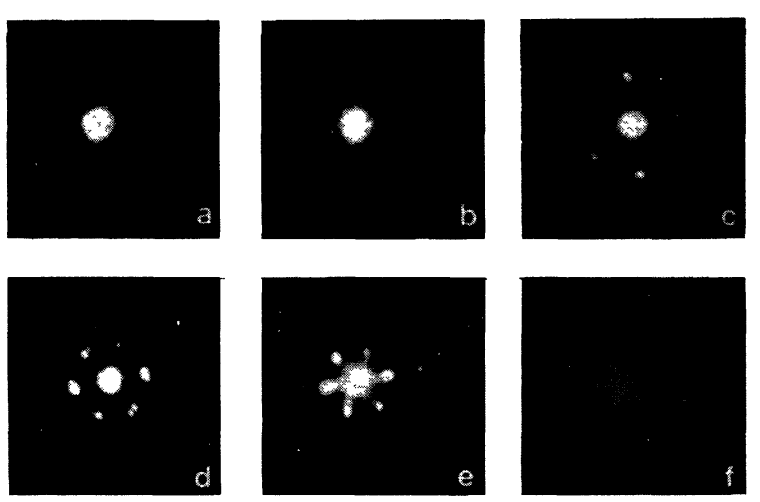

Fig. 6. - Typical thermal variation of incommensurate diffraction satellites around a $h k 0$ spot (with $h, k \neq 0$ ). a) $T \sim T_{\mathrm{i}}+1{ }^{\circ} \mathrm{C}$;b) $T \sim T_{\mathrm{i}}-0.5^{\circ} \mathrm{C}$; c) $T \sim T_{\mathrm{i}}-1.8^{\circ} \mathrm{C}$; d) $T \sim T_{\mathrm{i}}-2.8^{\circ} \mathrm{C}$; e) $T \sim T_{\mathrm{i}}-3.2^{\circ} \mathrm{C}$; f) $T \sim T_{\mathrm{i}}-3.7^{\circ} \mathrm{C}$. 
agreement with neutron and X-ray diffraction data, systematic extinctions are observed around $h 00$ type spots, of satellites with $\mathbf{k}$ along $\mathbf{a}^{*}$ (and symmetry related positions). In fact, as announced in section 2 , a deviation of $\mathbf{k}$ from the pure crystallographic directions can be expected (and has been observed in quartz). By displacing the sample under the beam, it is indeed possible to observe a splitting of the satellites which no longer lie exactly along the $\mathrm{OY}$ axis when the temperature is lowered. There are therefore regions, with opposite rotations $\pm \varepsilon$ of $\mathbf{k}$ around the $z$ axis.

On cooling from the $\beta$ phase, one first observes a diffuse scattering in the $O Y$ directions (Fig. 6a), followed by the apparition of the satellites themselves. When $T_{\mathrm{c}}$ is approached, the satellites gradually lie closer to the central peak, whereas $\varepsilon$ increases and higher order satellites appear. In figure $6 \mathrm{e}$, the second order satellites at $\pm 2 \mathbf{k}_{i}$ and at $\mathbf{k}_{i} \pm \mathbf{k}_{j}$ $(i \neq j)$ are clearly observed; this figure indicates of course a spatial superposition of the three modulations. At the transition to the $\alpha$ phase, the diffraction pattern becomes complex : the first order satellites on both sides of the $O Y$ axis are no longer resolved as they lie close to the Bragg reflection. Moreover, there is a manifestation of disorder since radial rods are evident (Fig. 6f) (this situation corresponds to a superposition of anisotropic diffraction patterns as will be discussed in the next paragraph). Such a strong increase of the satellite width in the $\mathbf{k}$ direction, is commonly reported at lock-in transitions. These characteristics make the determination of $\varepsilon$ close to $T_{\mathrm{c}}$ difficult, but we shall see in the next paragraph that a measurement can be done on the pictures.

From the diffraction data, it is possible to get the thermal variation of $k$ and $e$, presented in figure 7a. $k$ decreases as soon as the temperature is lowered below $T_{\mathrm{i}}$, and then jumps from the value $k_{\min }=0.01 a^{*}$ to zero. The angular deviation in $\mathrm{AlPO}_{4}$, as in quartz, increases regularly on cooling. It is interesting to compare the thermal variation of $k$ in this type of sample, to the thermal variation which was determined by neutron scattering (and electron microscopy) with an A-crystal. In the former case, the wave vector begins to change in the close neighbourhood of $T_{i}$, whereas in the latter, there exists a plateau extending approximately on $1^{\circ} \mathrm{C}$. This observation is consistent with an absence of pinning (or much weaker effects) in the B-samples.

The $\varepsilon$-angle can be calculated in the framework of the phenomenological theory [16]: one gets $(\varepsilon=\varphi-\pi / 2)$

$$
\begin{aligned}
\cos (3 \varphi)=\left[4 \rho r_{1}\right. & \left.\mathrm{C}_{66} /(a k K)\right] \times \\
\times & {\left[1+\frac{2}{3} G k^{2} C_{11} /\left(a r_{1}\right)\right] . }
\end{aligned}
$$

Where

$$
K=C_{11}-C_{66}
$$

With another approach, Walker [24] has obtained an expression

$$
\varepsilon=C \frac{\int\left(d \eta / d X^{\prime}\right)^{3} d X^{\prime}}{\int\left(d \eta / d X^{\prime}\right)^{2} d X^{\prime}}
$$

where $C$ is a constant, $\eta$ the O.P., and the $\mathrm{O} X^{\prime}$-axis is perpendicular to the wall. This result is interesting because, within the domain approximation of the I phase ( $\eta$ is constant, with a value $\pm \eta_{0}$ in a region, and then jumps to $\mp \eta_{0}$ when one follows the modulation direction), it relates $\varepsilon$ to the width e of the walls :

$$
\varepsilon \simeq 2 C \eta_{0} / e
$$

$\eta_{0}$ increases below $T_{\mathrm{i}}$ and the walls should sharpen up : this expression is therefore in agreement with the measured $\varepsilon$ angles in quartz [17] and berlinite. More precisely, it can be used to estimate the shape of the thermal variation of $e$, since the temperature dependence of $\eta_{0}$ is known $[10,11]$ from the satellite intensity in $\mathrm{SiO}_{2}$ and $\mathrm{AlPO}_{4}$ :

$$
e \sim \eta_{0} / \varepsilon \sim \frac{\left(T_{\mathrm{i}}-T\right)^{1 / 2}}{\varepsilon(T)}
$$
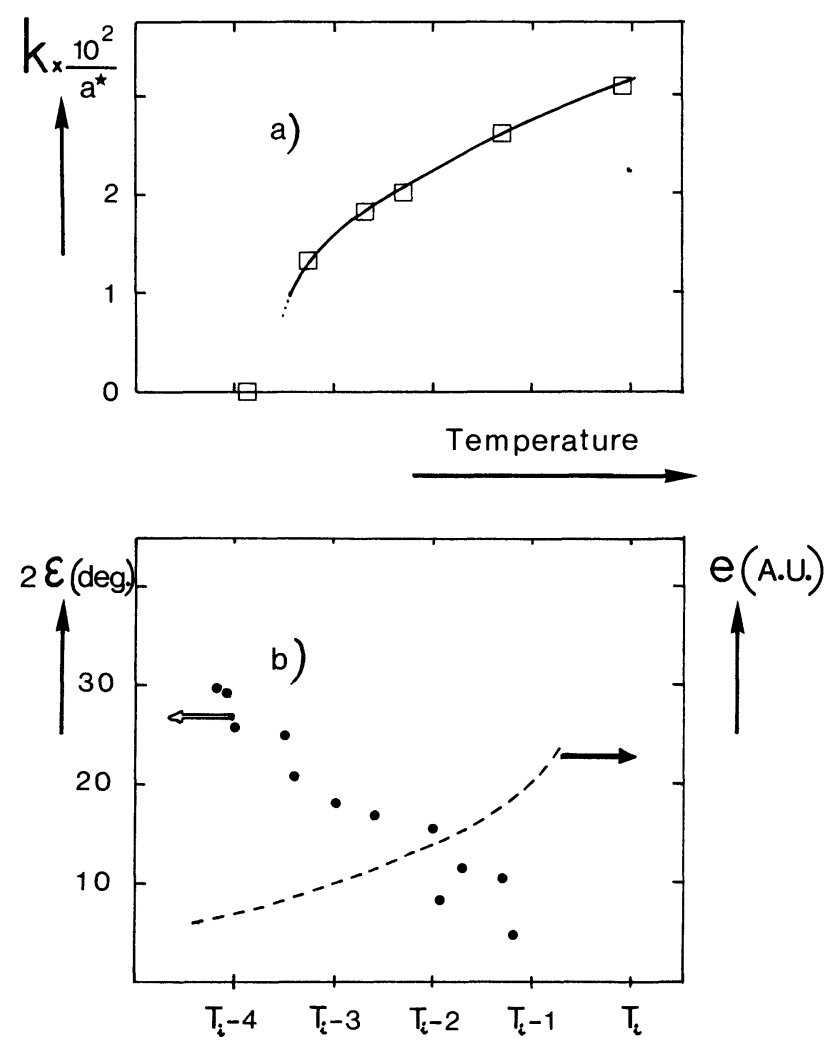

Fig. 7a. - Thermal variation of the modulation wave vector $k$ (electron diffraction data obtained with a berlinite B-sample). b) Temperature variation of $\varepsilon$-angle, and estimation of the wall width evolution (arbitrary units) from formula (7) (see text). 
The estimation of the thermal variation of $e$ is plotted in figure $7 \mathrm{~b}$. However one has to keep in mind the approximations used to deduce it : formula (5) can be approximated by (6) in the temperature range where the domain-texture describes well the incommensurate phase, namely when $e$ is small compared to the modulation wave length. When $e$ increases, this condition is satisfied with a poorer accuracy, as the modulation is expected to become sinusoidal close to $T_{\mathrm{i}}$. In particular, the estimation of $e$ is not valid close to this temperature, as the concavity of the curve has to be inversed : in the modulation regime, it is no longer possible to speak about domain texture, as the domain size reduces to zero, and $e$ tends to the constant value $\lambda / 2$ ( $\lambda$ is the modulation wave length $2 \pi / k) . \lambda$ is known from satellite position measurements, but the absolute scale for the values of $e$ cannot be deduced from our measurements, so that the comparison between $e$ and $\lambda / 2$ cannot be done; the temperature region in which the wave is approximately sinusoidal is therefore not exactly known. For this reason, only estimates valid close to $T_{\mathrm{c}}$, are represented in figure $7 \mathrm{~b}$. The increase of $e$ when $T$ approaches $T_{\mathrm{i}}$ has the signification of a change to the sinusoidal regime ; it is consistent with the observed simultaneous vanishing of higher order satellites.

\subsubsection{Direct space observations.}

a) $\varphi$-domains.

As already evidenced, the possible six orientations of the walls, determined by the angles with the $X$ axis $\varepsilon+n 2 \pi / 3$ and $-\varepsilon+n 2 \pi / 3(n=-1,0$, $+1)$, lead to the presence of two « macro »-domains $\left(\varphi_{+}\right.$and $\left.\varphi_{-}\right)$, corresponding to the former and latter angles respectively. Both domains are seen in figure 8 , and the interface between them is well resolved : its structure is in good agreement with Walker's analysis [24]. In some cases, it can be observed that small $\varphi_{ \pm}$-macrodomains included in a $\varphi_{\mp}$-region, were unstable : they regularly vanish and reappear (with the same shape), with a frequency about the $\mathrm{Hz}$ that slightly increases with the beam induced electric field. This dependence is likely to be related to ferroelectricity in the I-phase, as predicted by M. Vallade and B. Berge (reported in Ref. [23]) ; further theoretical investigations by Walker showed that the electric polarization should be carried by the walls themselves [25]. Energetic arguments, taking into account bulk and surface energies of the small domains can be invoked to explain the observed phenomena, but the " memory " effect on the position of the interface has an unclear origin. An interesting feature is the presence of intrinsic defects of the I phase, corresponding to dislocations in the domain-texture. Such defects were already invoked in the study of reference [5], but the detail of their structure was not clear. In their core we can observe that an orientation of wall corresponds to the other $\varphi$ macrodomain, so that these defects can be considered as small inclusions in a $\varphi_{+}\left(\right.$resp. $\left.\varphi_{-}\right)$macrodo-
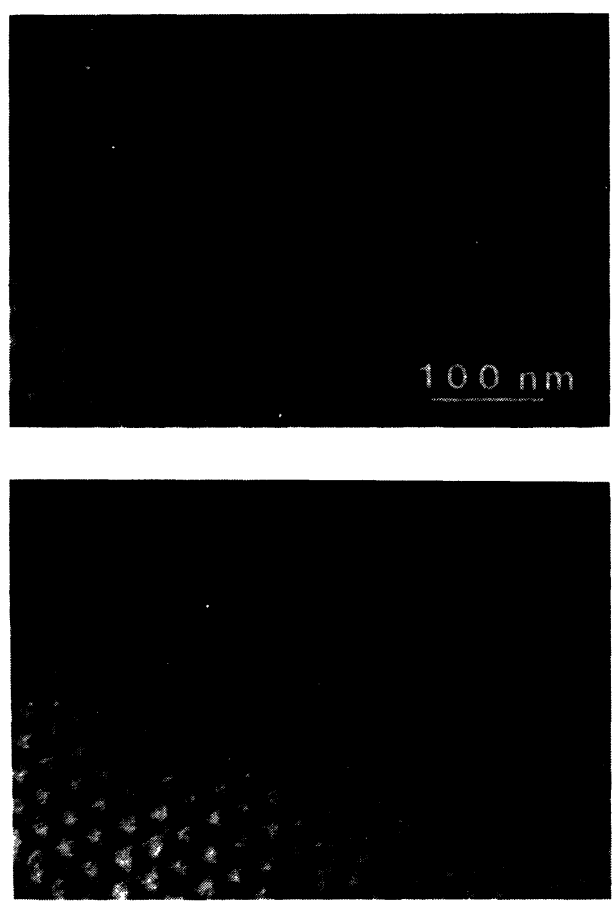

Fig. 8. - Bright field images of the I-phase in $\mathrm{AlPO}_{4}$ (Bsample) obtained at different times at a constant temperature. Note the change of the core of the defect indicated by an arrow.

main, of a $\varphi_{-}$(resp. $\varphi_{+}$) one. This is illustrated in figure 9. Of course, such defects are much more likely to occur than the distortions invoked in [5]: in the former case, the orientation of the walls is quasi everywhere that which corresponds to a minimum of energy, whereas in the latter, there is an energy cost due to the local rotation of walls in directions which are no longer perpendicular to the wave vectors.

b) P defects.

The other type of imperfections in the domain texture is related to the $\mathrm{P}$ defects observable perpen-

$$
k+\delta k
$$

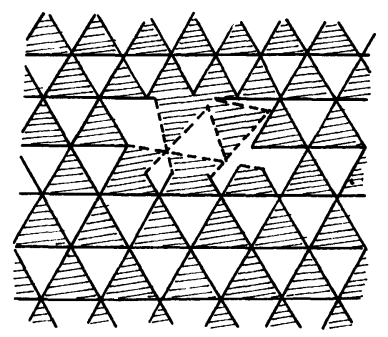

k

Fig. 9. - Schematic representation of the defect observed in figure 8. Hatched and full lines refer to walls corresponding to $\varphi_{+}$and $\varphi_{-}$macrodomains. 
dicularly to c. At a fixed temperature, these defects can also be created (by pairs) and then annihilated according to the process schematized in figure 10 (and a density of probability of type $w=\mathrm{e}^{-2 E(P) / k T}$, it can be shown by symmetry that both defects of a pair have the same energy cost $E(P))$. Both families of defects, $\mathrm{P}$ and $« \varphi$ » can in principle participate in the $\mathbf{k}$ variation in the I-phase. However, the observations performed when the beam is perpendicular to c, show a quick motion, along $\mathrm{OZ}$, of point-defects producing a variation from $k$ to $k+\delta k$ where $\delta k$ can be small (and depends on the P-defects density). We therefore believe that these point imperfections play a key role in the k-variation, not only in the crystals of the A type, but also in the B-samples.
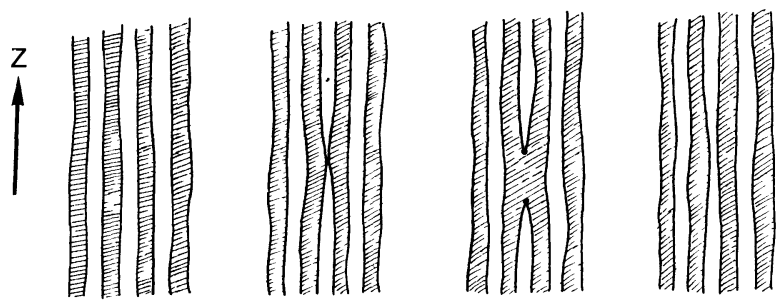

Fig. 10. - Schematic drawing according to video recording showing how fluctuations of incommensurate periodicity can create pairs of $P$ defects.

\section{c) Elongated triangles.}

At the lock-in, the transition could also be induced by the motion of the preceding deperiodization defects. However, the situation is complicated by the fact that the possibility of rotation of the walls by an angle $\pm \varepsilon$, is used by the system. Indeed, close to $T_{\mathrm{c}}$, an intermediate state characterized by elongated triangles (with the angles $2 \varepsilon, \pi / 3$, and $2 \pi / 3-2 \varepsilon$ ), takes place (Fig. 11a) (see also Ref. [14]) and can be sometimes well ordered, giving diffraction patterns as outlined in table. These objects are built with walls from both $\varphi$-macrodomains, as figure $11 \mathrm{~b}$. They are always observed at the lock-in transition, independently of the sense of temperature variation. They allow an extension of the measures of $\varepsilon$, in the whole temperature range. One finds for the maximum values of $\varepsilon$ : approximately $10^{\circ}$ in quartz and $15^{\circ}$ in the best berlinite crystals, but sometimes strong deviations $\left(\varepsilon=5^{\circ}\right.$ in some $\mathrm{AlPO}_{4}$ crystals). This sample dependence is likely related to a pinning of the walls by extrinsic defects, and supports the explanation of Van Landuyt $e t$ al. to account for the anomalies which were observed on the angles characterizing the " coarse » Dauphiné twins in the same material [14].

The creation of this new state is a way for changing drastically the distance between walls, locally and in one direction. The system does not therefore remove walls continuously (as expected e.g. in the phenomenological theory) but because of

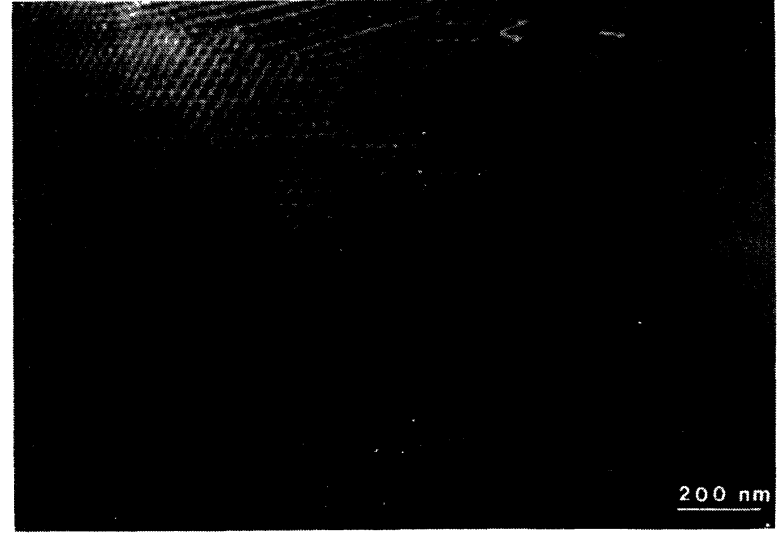

a)

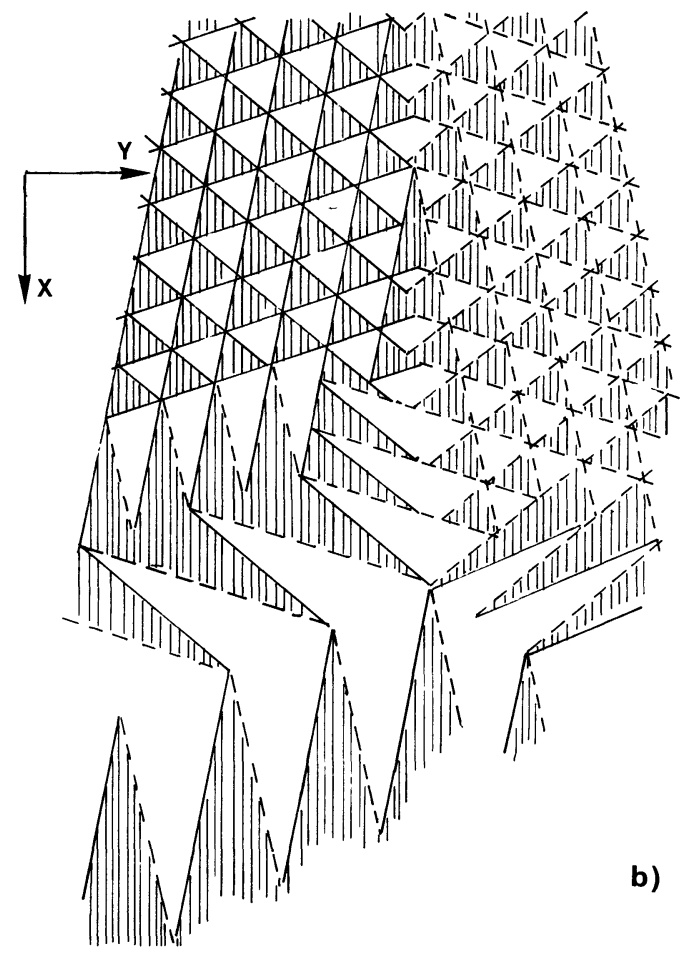

Fig. 11a. - Bright field image observed in $\mathrm{AlPO}_{4}$, showing the incommensurate and the intermediate phase in the presence of a thermal gradient. b) Structure of the $T_{\varepsilon}$-triangle textures. The $T_{\varepsilon}$ triangles are built using walls from $\varphi_{+}$and $\varphi_{-}$macrodomains (the same conventions as in Fig. 9 are adopted).

a pinning mechanism, it can be in supercooled and superheated states. As the lock-in transition is approached, the dynamical phenomena, already existing in the I-phase, are enhancing, and the vibrations of the walls become such that an hesitation occurs between two configurations: equilateral (E.T.) and elongated triangles $\left(T_{\varepsilon}\right)$, as shown in figure $12 \mathrm{a}$. This is outlined in figure $12 \mathrm{~b}$. The existence on cooling of the $(2 \varepsilon, \pi / 3,2 \pi / 3-2 \varepsilon)$ triangles $\left(T_{\varepsilon}^{\prime} \mathrm{s}\right)$ can be understood with energetic arguments : in the $\alpha$ (commensurate) phase far from $T_{\mathrm{c}}$, the walls have a positive energy mainly due to the term with the $g / 2$ coefficient in (1). As $\eta$ increases, 

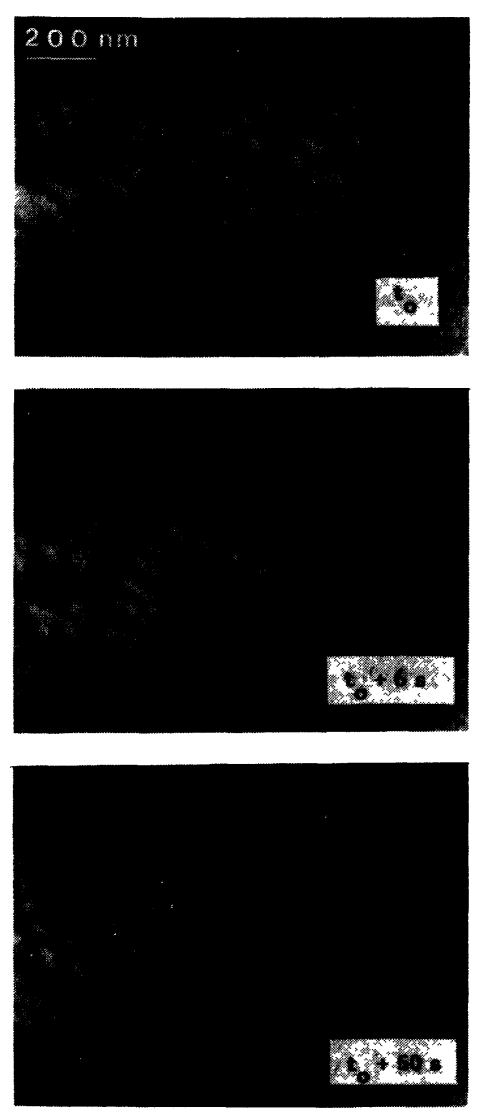

a)

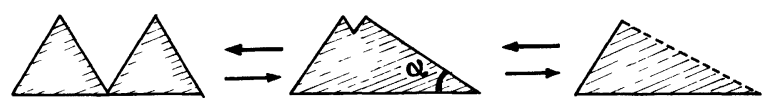

b)

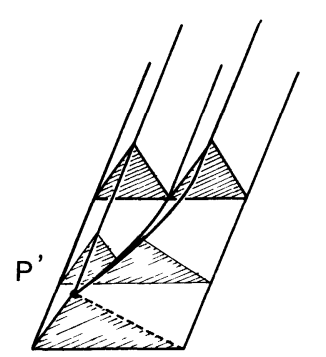

c)

Fig. 12a. - Dark field image 330 obtained with a quartz sample at constant temperature, showing the instability of the $T_{\varepsilon}$ triangles texture (from video recordings). b) Vibrational process of the passage from the $T_{\varepsilon}$-triangles to the E.T.'s (from video observations). c) Interpretation of the process presented in figure $12 \mathrm{~b}$, involving $\mathrm{P}^{\prime}$ defects.

the system tends to reduce the wall density in order to lower its free energy (Fig. 13). In the I-phase, the situation is reversed since because of the Lifchitzlike term in (1), the walls have now a negative free energy, so that the ground state corresponds to the regular wall lattice, with a density stabilized by
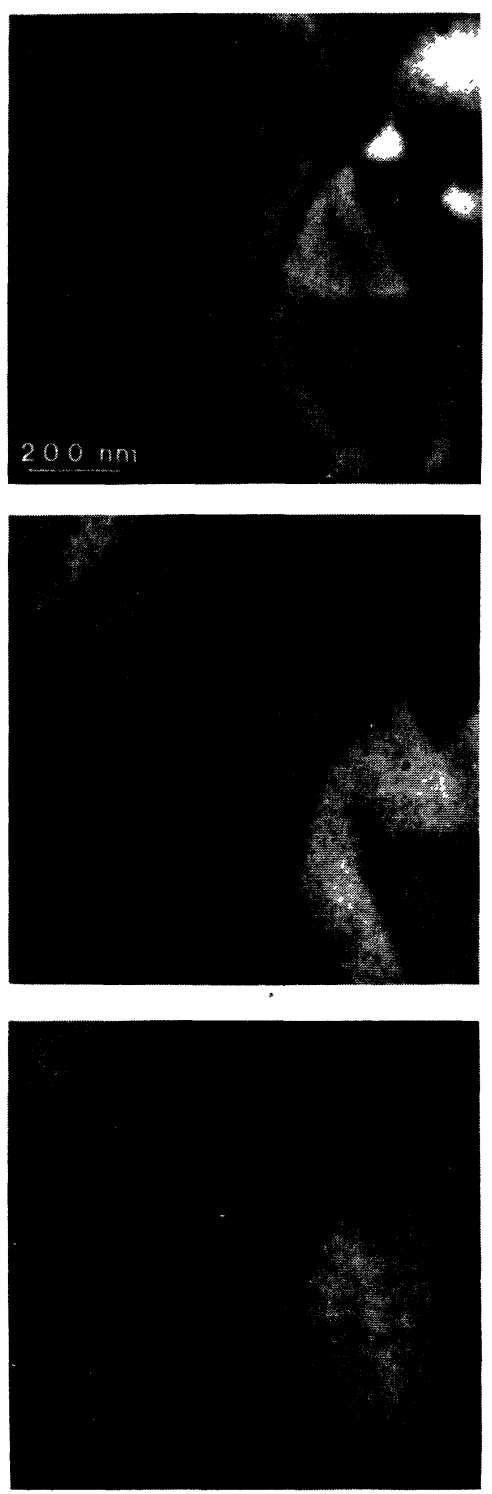

Fig. 13. - Bright field image of $\mathrm{AlPO}_{4}$ showing the decrease of the wall density when $T$ is lowered below $T_{\mathrm{c}}$.

repulsive (elastic) interactions between them. Let us call $T_{\mathrm{c}}^{0}$ the temperature at which the wall energy per unit area vanishes. If a frontal collective motion of the DC's is hindered, the system is at $T<T_{\mathrm{c}}^{0}$ still characterized by the E.T. configuration, and the decrease of the wall density can be in principle obtained in two different ways : 1 ) the motion in the $\mathrm{OZ}$-direction of $\mathrm{P}$ defects (e.g. introduced from the specimen boundaries). 2) the process outlined in figure $12 \mathrm{~b}$. As they are bounded by walls which have a smaller surface than the E.T.'s, the $T_{\varepsilon}$ 's become energetically more favourable below $T_{\mathrm{c}}^{0}$. Indeed, the energy versus the $\alpha$-angle (defined in Fig. 12b) presents two local minima, at $2 \varepsilon$ and $2 \pi / 3$, which change as a function of temperature, as shown in figure 14. It follows a variation of the probability of the $\pi / 3$ and $2 \varepsilon$ angles as $T$ is lowered. 


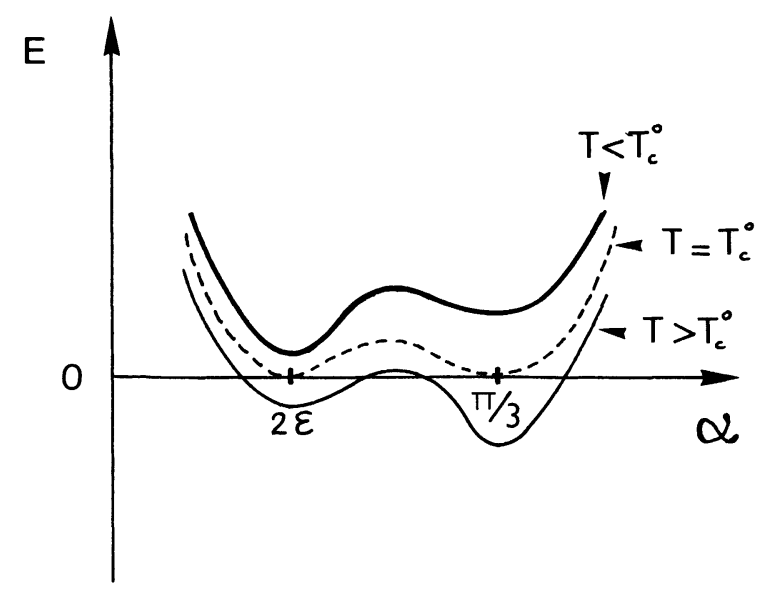

Fig. 14. $-\alpha$-dependence of wall energy at various temperatures ( $\alpha$ is defined in Fig. 12b).

At a given instant $t$, there is no reason for $\alpha$ to be constant along $\mathrm{OZ}$. The observed phenomena can therefore be due to some point defects $\mathrm{P}^{\prime}$ (see Fig. 12c) topologically equivalent to the P-ones, and the $z$-coordinate of which can fluctuate. This situation is supported by the observation in the geometry where the electron-beam is perpendicular to $\mathrm{OZ}$ (Fig. 15). The lock-in transition proceeds therefore as a classical nucleation [26] : a new phase appears because of the thermal fluctuations (Fig. 16), and the nucleus is stable when the energy cost for its creation (here that of the defects) is smaller than the gain in the bulk.

Apart from the homogeneous nucleation, the new phase. can be favoured by the presence of defects, and reciprocally the unstable phase can be maintained far from the transition point. We thus observed $T_{\varepsilon}$ 's of great size, pinned on the boundary of the sample at about $20^{\circ} \mathrm{C}$ below the lock-in transition temperature. The observations concerning defects and inhomogeneous nucleation will be the subject of a further paper.

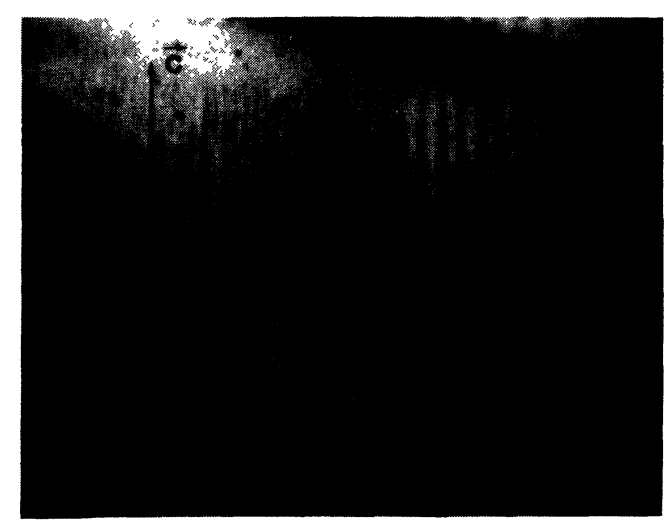

Fig. 15. - Bright field image obtained with a quartz sample. The electron beam is perpendicular to $\mathbf{c}$; the regular fringes on the left side of the picture correspond to the incommensurate phase, and the right side to the $T_{\varepsilon}$ triangles texture.
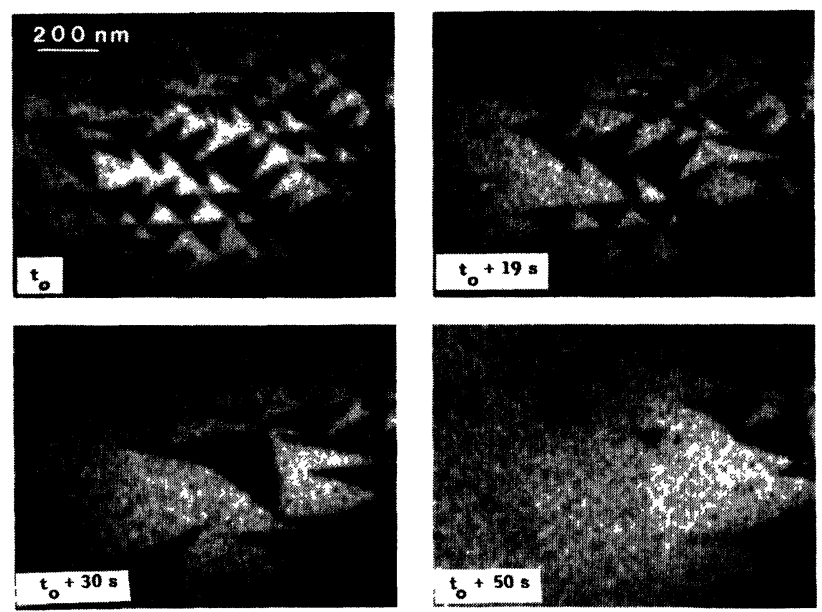

Fig. 16. - Dark field images 330 of quartz at a constant temperature, showing the decrease with time of the $T_{\varepsilon}^{\prime}$ density (from video).

\section{Conclusion and perspectives.}

Electron microscopy is a powerful tool for the study of incommensurate structures, and is unique for obtaining direct information.

In $\mathrm{AlPO}_{4}$, we have now a set of consistent data, since the phenomena observed by calorimetry $\left(C_{\mathrm{p}}\right)$ and optical methods $(\Delta n)$ can be well understood and related to the neutron diffraction results in this compound. Indeed, the wave vector variation is induced (in $\mathrm{SiO}_{2}$ and $\mathrm{AlPO}_{4}$ ) by a motion, along $\mathrm{OZ}$, of deperiodization defects. This mechanism leads us to assume that the DC's are not completely free to have a frontal motion. In samples with a high concentration of defects (such as the A berlinite crystals), the point defects intrinsic to the I phase can in turn be pinned or hindered in their motion, which results in the series of previously reported anomalies. All the new results shown here can be well understood qualitatively on the basis of existing theories [16, 24]. An earlier assumption [14] that pinning effects were responsible for the discarding from theory in $\mathrm{AlPO}_{4}$, is clearly confirmed.

In the case of incommensurate phases corresponding to a violation of the Lifchitz criterion, the phenomenological theory predicts a continuous lock-in transition, in contradiction with experiments ; the situation is changed by introducing some secondary order parameters, since the first order character can then be accounted for. However, there is also a suggestion [19], that a pinning of the walls can be responsible for the discontinuous change at $T_{\mathrm{c}}$ (or its enhancement), since it forces the system to be in supercooled and superheated states. In spite of a situation different for quartz and berlinite (where in particular a theoretical analysis of the order of the transition at $T_{\mathrm{c}}$ is lacking and where the discontinuous change is clearly marked), our study supports this assumption, as in relation with pinning, superheated and supercooled states were observed. 
Table I. - Schematic representation of the thermal evolution of the modulated phase.<smiles>C[13CH]</smiles>

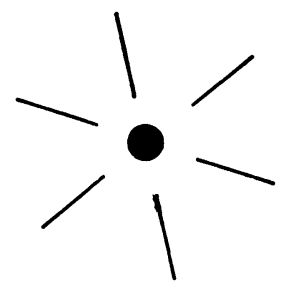

a) $T \geqslant T_{\mathrm{i}}$

Diffuse lines in $\mathbf{a}^{*}$ direction and equivalents.

No particular contrast in direct space observations.
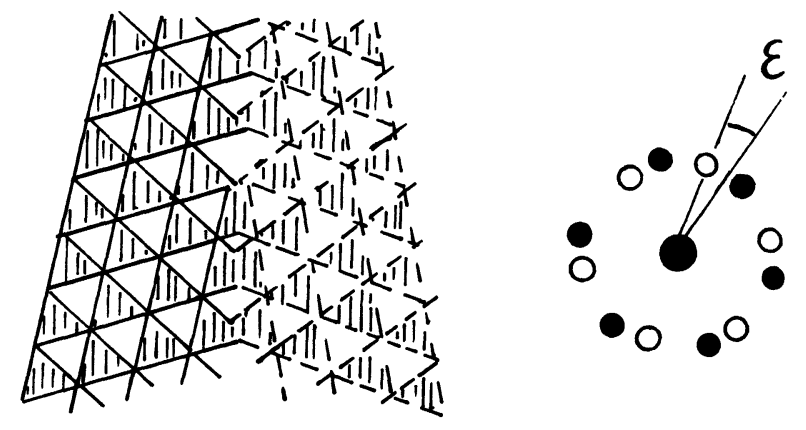

b) $T_{\mathrm{i}} \geqslant T>T_{\mathrm{c}}$

Satellite peaks at $\pm k$ in three directions deviated of an angle $+\varepsilon$ (or $-\varepsilon$ ) from the direction $\mathbf{a}^{*}$ and equivalents. Possibility to get the both satellites corresponding to $\pm \varepsilon$.

Microstructure consisting of regular equilateral triangles (E.T.). Possibility to obtain two macrodomains $\varphi_{ \pm}$(Fig. 8).

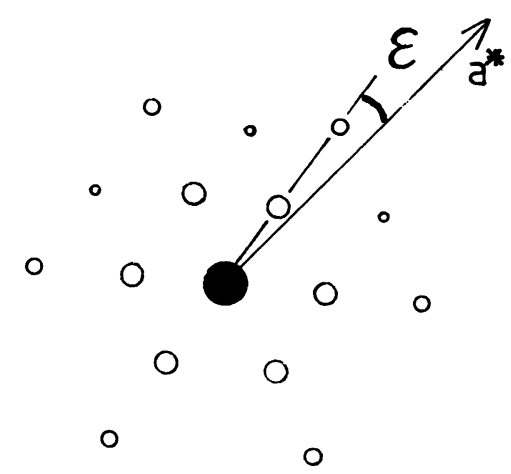

c) $T \geqslant T_{\mathrm{c}}$

Satellite peaks at $\pm n k(n=1,2)$ deviated from an angle $+\varepsilon$ (or $-\varepsilon)$ from the direction $\mathbf{a}^{*}$ and equivalents possibility to obtain :

- the both satellites $\pm \varepsilon$

- the third harmonic $(n=3)$.

Satellite peaks at $\mathbf{k}_{i}+\mathbf{k}_{j}(i \neq j)$.

Same observations as in $b$ ) with bigger equilateral triangles (Fig. 11, a).

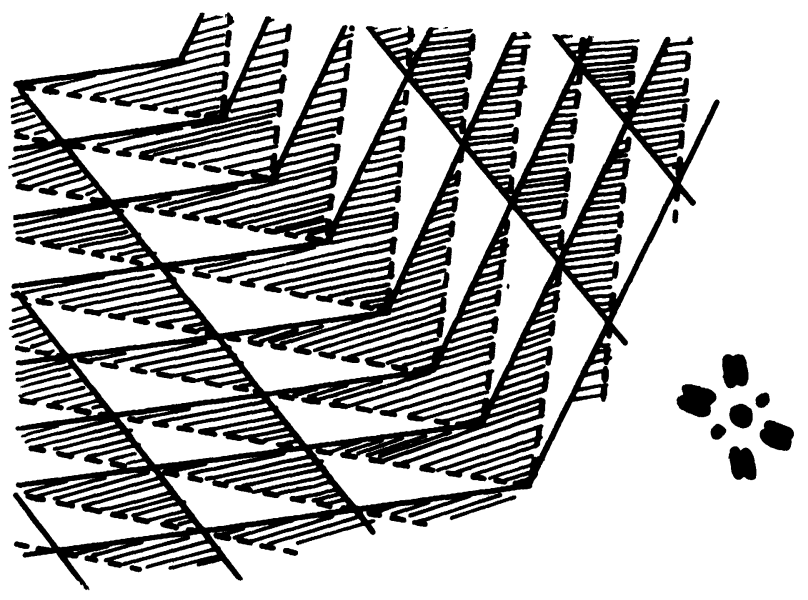

d) $T \leqslant T_{\mathrm{c}}$

Anisotropic diffraction pattern : two pairs of large satellite peaks and another pair with a $k$ value about twice smaller.

Elongated triangles $\left(T_{\varepsilon}\right)$ in a relatively good arrangement. 
The fact that the $k$-variation is always induced by the motion of the P defects, is interesting, and it is worth discussing this point. In other words, we can say that there is a nucleation, at each temperature, of a new I-phase with a wave vector changed by a quantity $\delta k$ which can be weak (and depends on the P-defect concentration). This experimental situation allows us to give an explanation of the memory effect, which differs from that of Jamet and Lederer [27], involving « defect density waves » (« DDW »). Indeed, let us assume that the temperature has been stabilized at a given value within the I-phase, at which $k=k_{0}$. As a result, the configuration of $\mathrm{P}$ defects has been quenched, and it is plausible to imagine that in the close neighbourhood of these objects, the defects of the crystal lattice have been modified. Therefore, in the crystal there exists centres of defects located at the positions of the $\mathrm{P}$ defects characterizing the $\mathbf{k}_{\mathbf{0}}$ vector. If the interaction energy between these centres and the deperiodization defects is sufficiently important, a plateau on the $k_{0}$ value is observed in the temperature interval where the P-defects are trapped by the centres.

In the same way, if the temperature is stabilized at several values in the I-phase, several sets of centres will be created, and will be further able to trap the configurations of $\mathbf{P}$ defects, thus inducing plateau's on the corresponding $k$-values. Let us recall that the memory effect, occurring in several incommensurate compounds, has been evidenced in quartz too [28]. In other incommensurate systems, it will be therefore interesting to examine the available experimental data in order to determine which mechanism holds in each case, pinning of DC's by DDW or trapping of deperiodization defects by « DLC's » (defects local centres).

In the present study we observed that close to the lock-in, the structure was changing with time, at a fixed temperature ; this mechanism, involving a progressive stabilization of the position of deperiodization defects, can of course hold in other incommensurate compounds presenting relaxation phenomena.
Our elucidation of the mechanism of the lock-in of the modulation wave vector $\mathbf{k}$ is in agreement with the ideas of Janovec et al. [19] and Prelovsek [20] since the transition proceeds as in a classical nucleation. When the temperature approaches the transition point, an enhancement of the vibrations of the walls and the nuclei is observed, with characteristic times of about $5 \times 10^{-2} \mathrm{~s}$. Further experiments are necessary to determine whether it corresponds to a specific frequency or to a wider spectral range.

\section{Remark.}

Recently, a controversy concerning incommensurate phase of quartz gave rise to comments by N. Kato and K. Gouhara [29] on the electron microscopy studies (Ref. [5] and [14]), and to a reply from the microscopists [30]. We think that most of the problems invoked in this controversy find an answer in the present work. In particular, the question of micro-twinning is a matter of terminology ; one starts with a sinusoidal wave close to $T_{\mathrm{i}}$, and harmonics of the displacement wave appear when $T$ approaches $T_{\mathrm{c}}$. In a forthcoming paper it will be shown that the E.T. contrasts can be interpreted without supposing a microtwin structure. Moreover, it is noteworthy that the features of the I phase of quartz and berlinite, as deduced by electron microscopy, are essentially the same as those obtained by neutron diffraction and can well be related to measurements by other methods.

\section{Acknowledgments.}

The berlinite crystals were grown in the ERA CNRS $\mathrm{N}^{\circ} 314$ at the University of Montpellier, by $\mathrm{A}$. Goiffon, J. Fourcade, J. C. Jumas and E. Philippot. We would like to thank them for providing samples. Thanks are also due to $M$. Combot-Marie for the quartz crystals. We acknowledge particularly Dr Hannu Mutka for fruitful discussions and pertinent remarks.

\section{References}

[1] Scott, J. F., Phys. Rev. B 4 (1971) 1360 ; Rev. Mod. Phys. 46 (1974) 83.

[2] Ng, H. N., Calvo, C., Can. J. Phys. 54 (1976) 638.

[3] Ecolivet, C., Poignant, H., Phys. Status Solidi (a) 63 (1981) K107.

[4] Aslanyan, T. A., Levanyuk, A. P., Pis'ma $Z h$. Eksp. Teor. Fiz. 28 (1978) 76 ; (Engl. transl. J.E.T.P. Lett. 28 (1978) 70 ; Solid State Commun 31 (1979) 547.

[5] Van Tendeloo, G., Van LanduYt, J., AmELINCKX, S., Phys. Status Solidi (a) 33 (1976) 723.

[6] Shapiro, S. M., Cummins, H. Z., Phys. Rev. Lett. 21 (1968) 1578.

[7] Shustin, O. A., Chernevitch, T. G., Ivanov, S. A., Yakovlev, I. A., Solid State Commun. 37 (1981) 65 (and other ref. therein).

[8] Levanyuk, A. P., Sannikov, D. G., Fiz. Tverd. Tela 18 (1976) 1927 (Engl. transl. Sov. Phys. Solid State 18 (1976) 1122).
[9] BAChHeimer, J. P., J. Physique Lett. 41 (1980) L559.

[10] Dolino, G., Bachheimer, J. P., Berge, B., ZeYen, C. M., J. Physique 45 (1984) 361.

[11] BAChHeimer, J. P., Berge, B., Dolino, G., SAINTGrégolre, P., ZEYEN, C. M., Solid State Commun 51 (1984) 55.

[12] Durand, J., Lopez, M., Cot, L., Retout, O., SaInT-Grégoire, P., J. Phys. C 16 (1983) L311.

[13] Saint-Grégoire, P., Schäfer, F. J., KleEmanN, W., Durand, J., Goiffon, A., J. Phys. C 17 (1984) 1375.

[14] Van Landuyt, J., Van Tendeloo, G., AmeLINCKX, S., WAlker, M. B., Phys. Rev. B 31 (1985) 2986.

[15] Yamamoto, N., Tsuda, K., Yagi, K., Japan J. Appl. Phys. 24 (1985) Suppl. 24-2, 811.

[16] Aslanyan, T. A., Levanyuk, A. P., Vallade, 
M., Lajzerowicz, J., J. Phys. C 16 (1983) 6705.

[17] Gouhara, K., Kato, N., J. Phys. Soc. Japan 53 (1984) 2177.

[18] Janovec, V., Phys. Lett. 99A (1983) 384.

[19] JANOVEC, V., GODEFROY, G. and L. R., Ferroelectrics 53 (1984) 333.

[20] Prelovsek, P., Ferroelectrics 54 (1984) 29.

[21] Golovko, V. A., Levanyuk, A. P., Zh. Eksp. Teor. Fiz. 77 (1979) 1556. (Engl. Transl. Sov. Phys. J.E.T.P. 50 (1979) 780.)

[22] Fousek, J., Kroupa, J., Japan J. Appl. Phys. 24 (1985) Suppl. 24-2, 787.

[23] Dolino, G., in Incommensurate Phases in Dielectrics, vol. II of Modern Problems in Condensed Matter
Sciences, Edited by R. Blinc and A. P. Levanyuk (North Holland, Amsterdam) 1985.

[24] Walker, M. B., Phys. Rev. B 28 (1983) 6407.

[25] Walker, M. B., Gooding, R. J., Phys. Rev. B 32 (1985) 7408.

[26] Landau, L., Lifchitz, E., Statistical Physics (pt 1) (Ed. Pergamon, Mir).

[27] Jamet, J. P., Lederer, P., J. Physique Lett. 44 (1983) L-257.

[28] Dolino, G., Japan J. Appl. Phys. 24 (1985) Suppl. 24-2, 153.

[29] Kato, N., Gouhara, K., Phys. Rev. B 34 (1986) 2001.

[30] Van Landuyt, J., Van Tendeloo, G., AmeLINCKX, S., Phys. Rev. B 34 (1986) 2004. 